\title{
Analysis of Channel Geometry and Sediment Transport in Palung and Chitlang Watersheds Using GIS
}

\author{
Bhola Nath Dhakal \\ Bhaktapur Multiple Campus, TU, Bhaktapur \\ E-mail:dhakalbn@gmail.com
}

\begin{abstract}
Channel geometry and sediment transport have been analyzed using Geographic Information System and statistical methods in Palung and Chitlang sub-watershed of Kulekhani watershed located in the Central Hills of Nepal, which covers 87.9 sq. km of land surface.

The study demonstrates that the channel geometry and sediment transport changes abnormally in downstream distance for both rivers, though there are some controlling factors i.e. lithology, land use, climate, vegetation cover etc.
\end{abstract}

Key words: Channel geometry, discharge, GIS, landscape, River morphology

\section{INTRODUCTION}

Nepal has high-energy environment because of seasonal monsoon precipitation, weak and active geology, and high relief but there is absolute lack of information on the channel geometry and river behavior in the context of Nepal. There is absolute lack of study on the relationship between hydraulic variables, which is essential in designing hydraulic structure and development of water resources. Water resources development or management represents an attempt to meet an existing or potential demand for water or related resources, such as energy and food (Alford, 1991). The study of the channel behavior is one of the most significant aspects of the water resources management.

Mountain streams are similar in their dynamics and form to stream in other parts of the World. Present environment is characterized by one of the high relief, tectonic activity and intensive land use provides great volumes of both water and sediment to the channels but does not force them to behave in an abnormal way. It is evident that the process in change in channel hydraulics varies with time and space (Caine and Mool, 1980).
River channel is the most dynamic component of the landscape. Dramatic change in channel morphology and river behavior occurs as a result of upstream control due to the change in climate and land use, and downstream influences due to change in base level. The relief, lithology, structure, climate and hydrology as well as vegetation cover also controls the actual erosion rate in an area (Chorley et al, 1984).

River hydraulics is a comprehensive study of the behavior of flow in natural channels, which require the knowledge of hydrology, geomorphology, and sediment transportation etc (Chow, 1986).

Hydraulic Geometry is the analysis of the interdependence of hydraulic variables of stream channels, where, channel width, depth, slope, velocity, sediments or particle's size, channel roughness and stream discharges are studied (Leopold, 1993).

The sediment loads of Himalayan Rivers are amongst the highest in the World, resulting in problems such as the siltation of reservoirs, blockage 
of river channels, water pollution and degrading of aquatic environment. In a rain-fed river system, the major sources of sediments are considered to be landslides and intensively cultivated hill slopes but little is known about the characteristics of the sediments from these sources, movements into the river system or transport down to the rivers (Johnson and Collins, 1996)

The channel morphology and river behavior is controlled by the relief, lithology, geology, climate and land use and land cover. Relief is an index of potential energy and lithological variation determine the erodibility of the surface materials and infiltration capacity. The geology controls the pattern of drainage and the climate controls both the runoff and vegetation. River hydraulics is a comprehensive study of the behavior of flow in natural channels, which require the knowledge of hydrology, geomorphology, and sediment transportation etc.

Stream channels are among the most dynamic components of the landscape. Through them, energy and mass are transferred from one location to another. It is evident that the process in change in channel hydraulics varies with time and space. This study aims to evaluate the similarity and differences in different variables of channel geometry of Palung and Chitlang Rivers as compared to other areas. The general objective of this paper is to analyze the hydraulic geometry and sediment transport of river channels in terms of river morphology. The specific objectives are;

i. To study the relationship among the hydraulic variables of channels and variation of these variables from one place to another and from one river to another.

ii. To study the change in channel behavior in down stream direction and their controls. iii. To discuss the type of sediments transported and deposited by streams in downstream direction.

\section{THE STUDY AREA}

The Palung and Chitlang River basins are located in southwest of Kathmandu Valley within the outer Himalayan Mountain. It covers the surface drainage area of 87.9 sq. km (65.1 sq. km of Palung River basin and $22.8 \mathrm{sq}$. km of Chitlang River basin). These are the sub-catchments of Kulekhani watershed. Geographically it is extended from $85^{\circ}$ $01^{\prime} 15^{\prime \prime}$ East to $85^{\circ} 12^{\prime} 30^{\prime \prime}$ East longitude and $27^{\circ}$ $35^{\prime} 00^{\prime \prime}$ North to $27^{0} 41^{\prime} 35^{\prime \prime}$ North latitude. Figure 1 shows the location map of study area.

The elevation of the watersheds ranges from 1510 $\mathrm{m}$ to $2540 \mathrm{~m}$ above mean sea level. The length of main channel of Palung River is $15.953 \mathrm{~km}$ and Chitlang River is $9.730 \mathrm{~km}$. The drainage density of Palung watershed is $5.084 \mathrm{~km} / \mathrm{km}^{2}$ and Chitlang is $4.119 \mathrm{~km} / \mathrm{km}^{2}$, and drainage frequency is $14 \mathrm{No} /$ $\mathrm{km}^{2}$ in Palung and $8 \mathrm{No} / \mathrm{km}^{2}$ in Chitlang basin.

The mean annual temperature of this area is $17.9^{\circ} \mathrm{C}$ and an average annual precipitation is about $1635 \mathrm{~mm}$. The drainage area comprises different land use categories i.e. forest, cultivation, shrub land, settlement, grass land, sand and water bodies. Cultivated land constitutes the largest land use categories. In Palung basin, it covers $54 \%$ of total basin area and forest area covers $34 \%$ of the total basin area. In Chitlang basin forest area comprises the largest land use category; it covers $60 \%$ of the total basin area. Similarly cultivated land covers $35.5 \%$ of the total basin area.

According to slope categories, about $46 \%$ of total basin area is under the moderate slope $\left(5^{\circ}-30^{\circ}\right)$ and $35 \%$ area is under steep slope (more than $30^{\circ}$ ) of the study area. Climatologically, $63 \%$ of total basin area is under warm temperate zone and $37 \%$ area is under cool temperate zone. 


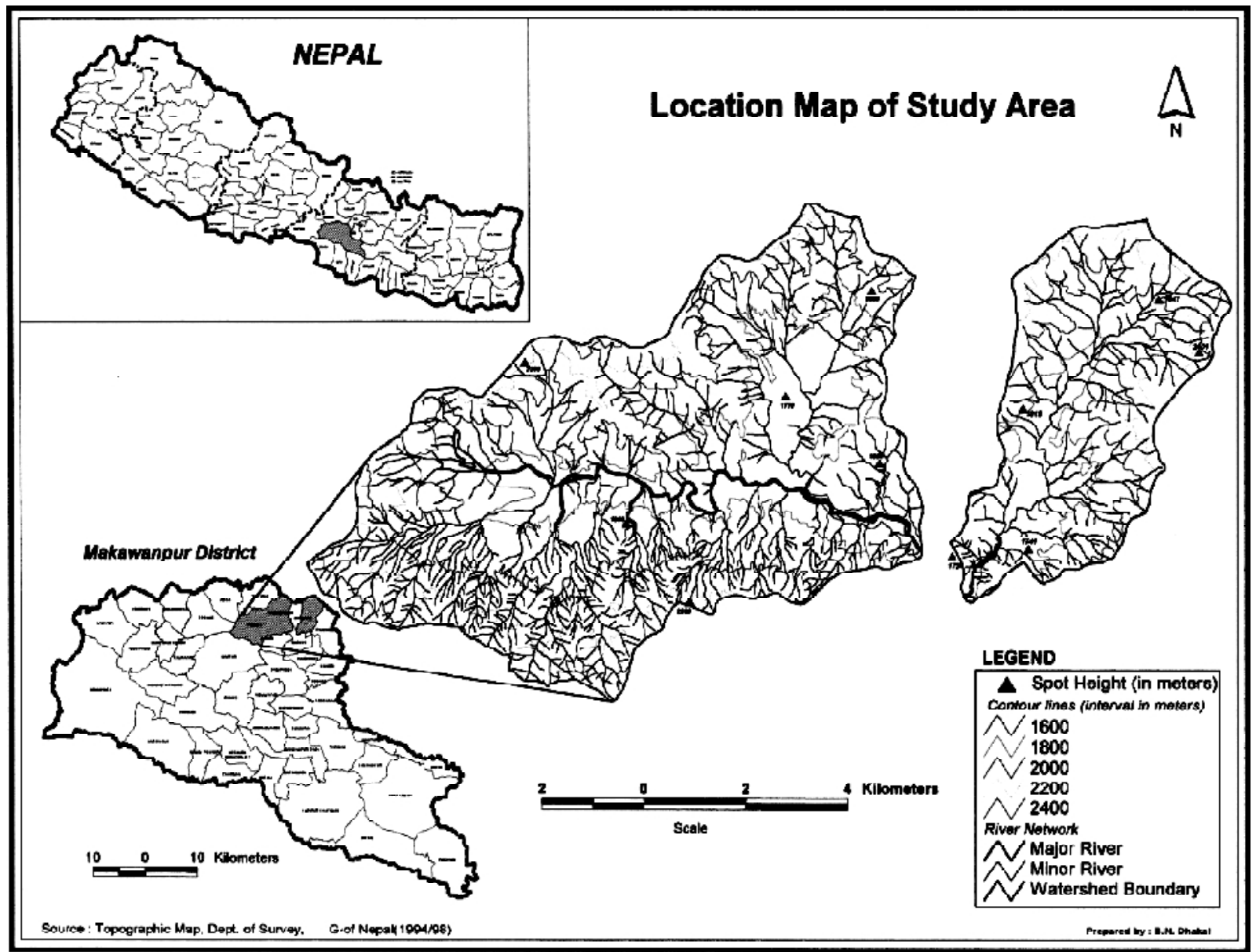

Figure 1: Location map of study area

\section{METHODOLOGY}

The collected data and information were analyzed by using different statistical formula, Arc INFO 3.5.1 and Arc View 3.2 Geographic Information System (GIS) software and other Computer Software programs. Beside these, cartographic and descriptive methods were also adopted.

For the flow hydraulics, some parameters tractive force or shear stress, stream power, stream power per unit area, Froude number and Reynolds numbers for both channels in each site have been calculated. The regression model was used to define the interrelationship of variables in downstream distance and stream slope with particles diameter. Correlation coefficients were tested at significance level $(\alpha)=0.01$. To define the shape factor, Sphericity $(\psi)$, Flatness $(\mathrm{Fw})$ and Elongation (Es) values were calculated based on three diameters of the sediment measured in each survey site along both channels. Similarly the regression was used to define the interrelationship of shape factors. The channel flow parameters and sediment parameters are calculated using the following equations.

i. $\quad$ Channel Capacity $(\mathrm{C})=\mathrm{WxR}$ where $\mathrm{W}=$ width of channel (m) $\mathrm{R}=$ mean depth (as hydraulic radius) $(\mathrm{m})$

ii. Stream discharge $(\mathrm{Q})=0.85 \times \mathrm{A} \times \mathrm{v}$ where $A=$ cross-sectional area $\left(\mathrm{m}^{2}\right)$ $\mathrm{v}=$ mean velocity $(\mathrm{m} / \mathrm{sec})$ 
iii. Tractive share stress $\left(\mathrm{T}_{\mathrm{c}}\right)=\gamma \mathrm{ds}$ where $\gamma=$ weight of water $\left(1 \mathrm{Nm}^{-3}\right)$ $\mathrm{d}=$ mean depth $\mathrm{s}=$ slope

iv. Stream power $\left(\mathrm{W}_{\mathrm{c}}\right)=\gamma \mathrm{dsv}$

where $\gamma=$ tractive force

$\mathrm{d}=$ depth of water $\mathrm{s}=$ slope

$\mathrm{v}=$ mean velocity.

v. Froude Number $(\mathrm{Fr})=\mathrm{v} /(\mathrm{gd})^{1 / 2}$ where $\mathrm{v}=$ water velocity $\left(\mathrm{ms}^{-1}\right)$

$\mathrm{g}=$ gravitational constant $\left(9.80665 \mathrm{~ms}^{-2}\right)$

$\mathrm{d}=$ water depth $(\mathrm{m})$

vi. Reynolds numbers $(\mathrm{Re})=\mathrm{du} * / \mathrm{v}$ where $\mathrm{d}=$ particle size $(\mathrm{cm})$

$u^{*}=$ shear velocity $(\gamma / \rho)^{1 / 2}$

$\rho=$ water density

$\mathrm{v}=$ Kinemetic viscocity

$$
\left(0.013 \mathrm{~cm}^{2} \mathrm{~s}^{-1}\right)
$$

vii. Sphericity $(\psi)=\left(B C / A^{2}\right) 1 / 3$

viii. Flatness $(\mathrm{Fw})=(\mathrm{A}+\mathrm{B}) / 2 \mathrm{C}$

ix. Elongation $(\mathrm{Es})=\mathrm{B} / \mathrm{A}$

where $A=$ Longaxis length of sediment $(\mathrm{mm})$

$\mathrm{B}=$ Intermediate length of sediment $(\mathrm{mm})$

$\mathrm{C}=$ Short axis length of sediment $(\mathrm{mm})$

\section{NATURE AND SOURCES OF DATA}

This study is based on both primary and secondary sources of data. Primary data were collected from the field measurement. Field survey was carried out for 21 days during January-February, 1997 to obtain data on hydraulic variables. Channel width, depth, stream slope, water discharge and particle size were measured by using simple instruments and method like measuring tape, Abney label, scale, stopwatch, cockball etc. For the discharge measurement float method was adopted. Similarly, sediment deposited along the River course was sampled for the sediment survey, where 30 particles (boulders) with different size were taken randomly for measurement in each survey station. In this study, the diameter $(\mathrm{a}=$ longest, $\mathrm{b}=$ intermediate and $\mathrm{c}=$ shortest) of 20 particles deposited by recent flows at a station and of 10 particles that had moved in the past were measured. Measured values are presented on the table 1 and 2 under the column iv, $\mathrm{v}$, vii, $\mathrm{x}$ and xi respectively. ARC INFO 3.5.1 and Arc View 3.2 GIS software was used to generate data on basin characteristics like stream distance, basin area, channel width and depth ratio, and map layers like geology, contour, rivers, land use, climate and slope. Statistical formula was applied to calculate channel capacity, velocity, tractive force, stream power, Froude number, Reynolds number and presented on table 1 and 2. Basic geometric properties of drainage system have been explored according to the devices formulated by Horton and Strahler (Strahler, 1968).

For the secondary information, Hydrometeorological data were taken from the Climatological Records (1971-1993) of Nepal (DHM, 1997) and Stream Flow Records (19631978) of Nepal (DHM, 1997), published, by the Department of Hydrology and Meteorology, Government of Nepal.

Information on drainage network, relief and land use pattern were compiled from the topographic sheets prepared by Department of Survey, Government of Nepal (1994/98) at the scale of 1:25000. Geological map prepared by the Department of Mines and Geology (1984) at the scale of 1:250000 were used to identify geology of study area and Land Capability Map prepared by the Land Resources Mapping Project(LRMP, 1978/ 82) at the scale of 1:50000 were used to extract slope and climate zone of study area. Besides these, various documents and reports were collected and reviewed. These maps were digitized using GIS software and necessary data were generated.

The hydraulic geometry and hydraulics of flow in Palung and Chitlang Rivers are presented in Table 1 and 2 respectively. Table 3 presents morphometric parameters for both rivers. 
Table 1: Hydraulic Geometry and Hydraulics of flow in Palung River.

\begin{tabular}{|c|c|c|c|c|c|c|c|c|c|c|c|c|c|c|}
\hline Station & $\begin{array}{c}\text { Distance } \\
(\mathbf{k m})\end{array}$ & \begin{tabular}{|l|} 
Basin \\
Area \\
$\left(\mathrm{km}^{2}\right)$ \\
\end{tabular} & \begin{tabular}{|l|} 
Channel \\
Width \\
W(m) \\
\end{tabular} & \begin{tabular}{|c|} 
Mean \\
Depth \\
R(m) \\
\end{tabular} & W/R & $\begin{array}{c}\text { Slope } \\
\mathbf{S}\left({ }^{\circ}\right)\end{array}$ & $\begin{array}{c}\text { Channel } \\
\text { Capacity } \\
\mathrm{C}\left(\mathrm{m}^{2}\right) \\
\end{array}$ & $\begin{array}{l}\text { Velocity } \\
\text { V(m/sec) }\end{array}$ & $\begin{array}{l}\text { Discharge } \\
\mathrm{Q}\left(\mathrm{m}^{3} / \mathrm{sec}\right)\end{array}$ & \begin{tabular}{|c|} 
Particle \\
Size \\
F $(\mathrm{cm})$ \\
\end{tabular} & \begin{tabular}{|c} 
Tractive \\
Force \\
$\left(T_{c}\right)$ \\
\end{tabular} & $\begin{array}{c}\text { Stream } \\
\text { Power } \\
\left(\mathbf{W}_{\mathrm{c}}\right) \\
\end{array}$ & $\begin{array}{c}\text { Froude } \\
\text { Number } \\
\text { (Fr) } \\
\end{array}$ & $\begin{array}{c}\text { Reynolds } \\
\text { Number } \\
(\mathrm{Re}) \\
\end{array}$ \\
\hline $\bar{A}$ & 091 & 2.713 & 1.2 & 0.1667 & 7.1986 & 0.675 & 0.2000 & 0.4400 & 0.0750 & 58 & 0.1127 & 0.0496 & 0.3438 & 15.130 \\
\hline $\bar{B}$ & 772 & 4.711 & 1.9 & 0.1170 & 16.2393 & 0.760 & 2223 & 5000 & 130 & 49 & .0889 & 0534 & 5601 & .252 \\
\hline$\overline{\mathrm{C}}$ & 405 & 4.948 & 2.6 & 0.1160 & 22.4138 & 1.027 & 3016 & 4615 & 1180 & 47 & 1191 & 0550 & 4327 & 2.604 \\
\hline D & 982 & 5.287 & 4.5 & 0.1257 & 35.7995 & 0.424 & 5656 & 3478 & 1670 & 43 & 0534 & 0186 & 3129 & 7.721 \\
\hline E & 579 & 5.552 & 3.7 & \begin{tabular}{|l|l|}
0.1769 \\
\end{tabular} & 20.9158 & 0.991 & 6540 & 3051 & 1690 & 34 & 1754 & |0535 & 2316 & 1.065 \\
\hline $\bar{F}$ & 892 & 5.716 & 3.8 & \begin{tabular}{|l|l|}
0.1543 \\
\end{tabular} & 24.6273 & 0.824 & 8863 & 143 & 600 & 32 & 1269 & 0399 & 558 & 8.85 \\
\hline$\overline{\mathrm{G}}$ & 113 & 5.794 & 4.0 & 1050 & 38.0952 & 781 & 4200 & 4348 & 600 & 34 & 0820 & \begin{tabular}{|l|}
0357 \\
\end{tabular} & 4285 & 7.56 \\
\hline$\overline{\mathrm{H}}$ & 486 & 8.889 & 8.0 & 0.0700 & 114.2857 & .257 & 5600 & 4400 & & 39 & 880 & 0387 & 5312 & 8.99 \\
\hline I & 25 & \begin{tabular}{|l|}
17.01 \\
\end{tabular} & 8.5 & 0.0671 & 126.6 & 1.600 & 04 & & & 38 & & 514 & 915 & 9.66 \\
\hline $\mathrm{J}$ & 78 & 17.16 & 6.8 & 0.1619 & 42.0012 & 1869 & 1009 & 2408 & & 40 & 408 & 0340 & 1905 & 1.66 \\
\hline $\mathrm{K}$ & 01 & 18.34 & 4.9 & 0.1628 & 30.0983 & 1.402 & 977 & 3792 & 71 & 37 & 2285 & 0866 & 3000 & 13.74 \\
\hline $\bar{L}$ & 242 & 18.53 & 5.8 & 0.0900 & 64.4444 & 1.271 & 5220 & 524 & 50 & 34 & 1144 & .0632 & 5880 & 8.936 \\
\hline M & 494 & \begin{tabular}{|l}
19.43 \\
\end{tabular} & 5.0 & 0.1189 & 42.0521 & 0.754 & 5945 & 5263 & 2659 & 35 & 0897 & .0472 & 4872 & 8.145 \\
\hline $\mathrm{N}$ & 801 & 19.65 & 7.0 & 0.1467 & 47.7164 & 1.213 & 0269 & & 2887 & 36 & 0.1783 & 0.0590 & .2754 & 11.81 \\
\hline $\bar{O}$ & 296 & 20.12 & 5.8 & 0.1478 & 39.2422 & 0.9 & 772 & & 880 & 3 & 67 & .0539 & 049 & 11.01 \\
\hline$\overline{\mathrm{P}}$ & 398 & 20.57 & 6.3 & 0.0928 & 67.8879 & 0.9 & 5846 & 5479 & & 3 & 376 & .0568 & 6784 & 7.81 \\
\hline Q & 259 & 20.82 & 6.5 & 0.1017 & 63.9 & 1.3 & 6612 & 06 & 20 & 35 & 379 & .0814 & 5905 & 0.05 \\
\hline $\mathrm{R}$ & 886 & 27.86 & 6.7 & 0.1273 & 52.6 & 1.150 & 529 & & 570 & 37 & 461 & 0.0941 & 5768 & 0.98 \\
\hline $\bar{S}$ & 281 & 31.24 & 5.8 & 0.1406 & 41.2518 & 1.351 & 155 & 813 & 7720 & 32 & 0.1905 & 0.1298 & .5793 & 10.853 \\
\hline $\bar{T}$ & 884 & 31.71 & 4.0 & 0.2925 & 13.6752 & 0.991 & 1.1700 & 4800 & 4800 & 30 & 0.2904 & 0.1394 & .2832 & 12.562 \\
\hline U & 673 & 32.11 & 10.6 & 0.0914 & 115.9737 & 2.824 & 0.9688 & 0.6131 & 5049 & 33 & 0.2570 & 0.1576 & 0.6490 & 12.999 \\
\hline $\mathrm{V}$ & .198 & 59.24 & 5.6 & 0.2513 & 22.2841 & 1.130 & 1.4070 & 385 & 0.6439 & 46 & 0.2836 & 0.1527 & .3432 & 19.03 \\
\hline$\overline{\mathrm{W}}$ & .529 & 59.48 & 9.2 & 0.1886 & 48.7805 & 0.795 & 1.7350 & 717 & 5481 & 54 & 1503 & 0.0559 & 2730 & 16.26 \\
\hline $\bar{X}$ & .151 & 59.77 & 8.7 & 0.2038 & 42.6 & 0.824 & 726 & & & 36 & & 0.0617 & 5595 & 11.46 \\
\hline $\bar{Y}$ & .391 & 59.92 & 6. & 0.1930 & 32.1 & 0.8 & & & & 37 & 519 & 0.0866 & 880 & 11.56 \\
\hline $\bar{Z}$ & .693 & 61.00 & 9.2 & 0.3059 & 30.0752 & 1.280 & 2.8143 & 0.2704 & 6468 & 20 & 917 & 0.1059 & .1561 & 13.61 \\
\hline $\mathrm{A}^{\prime}$ & 15.244 & 64.88 & 9.7 & 0.1436 & 67.5487 & 1.235 & 1.3926 & 800 & 6866 & 29 & 17778 & 0.1031 & 4881 & 9.50 \\
\hline $\mathrm{B}^{\prime}$ & 15.635 & 65.05 & 4.8 & 0.3538 & 13.5669 & 0.933 & 1.6980 & 0.4694 & 6770 & 19 & 3303 & 0.1550 & .2519 & 8.48 \\
\hline$\overline{\mathrm{C}}$ & \begin{tabular}{l|l}
15.953 \\
\end{tabular} & 65.10 & 4.5 & 0.2895 & 15.5440 & 0.713 & 1.3028 & 0.5800 & 0.6423 & 10 & 0.2068 & 0.1199 & 0.3439 & 3.534 \\
\hline
\end{tabular}


Table 2: Hydraulic Geometry and Hydraulics of flow in Chitlang River.

\begin{tabular}{|c|c|c|c|c|c|c|c|c|c|c|c|c|c|c|}
\hline Station & $\begin{array}{c}\text { Distance } \\
(\mathbf{k m})\end{array}$ & $\begin{array}{l}\text { Basin } \\
\text { Area } \\
\left(\mathrm{km}^{2}\right)\end{array}$ & $\begin{array}{l}\text { Channel } \\
\text { Width } \\
\text { W(m) }\end{array}$ & $\begin{array}{l}\text { Mean } \\
\text { Depth } \\
\text { R(m) }\end{array}$ & $\mathbf{W} / \mathbf{R}$ & $\begin{array}{c}\text { Slope } \\
\mathbf{S}\left({ }^{\circ}\right)\end{array}$ & $\begin{array}{c}\text { Channel } \\
\text { Capacity } \\
\mathrm{C}\left(\mathrm{m}^{2}\right)\end{array}$ & $\begin{array}{l}\text { Velocity } \\
\mathrm{V}(\mathrm{m} / \mathrm{sec})\end{array}$ & $\begin{array}{l}\text { Discharge } \\
\mathbf{Q}\left(\mathrm{m}^{3} / \mathrm{sec}\right)\end{array}$ & $\begin{array}{c}\text { Particle } \\
\text { Size } \\
\text { F (cm) }\end{array}$ & $\begin{array}{l}\text { Tractive } \\
\text { Force } \\
\left(\mathbf{T}_{\mathrm{c})}\right.\end{array}$ & $\begin{array}{l}\text { Stream } \\
\text { Power } \\
\left(W_{c}\right)\end{array}$ & $\begin{array}{c}\text { Froude } \\
\text { Number } \\
\text { (Fr) }\end{array}$ & $\begin{array}{c}\text { Reynolds } \\
\text { Number } \\
\text { (Re) }\end{array}$ \\
\hline $\bar{A}$ & 1.886 & 1.625 & 1.2 & 0.0580 & 0.6897 & 0.445 & 0.070 & 0.4055 & 0.0240 & 31 & 0.0258 & 0.0105 & 0.5377 & 3.869 \\
\hline B & 652 & 928 & 1.8 & 0.1280 & 1.0625 & 0.754 & 230 & 5263 & 030 & 29 & 0965 & 0508 & 4698 & \\
\hline $\mathrm{C}$ & 146 & 040 & 2.4 & 1360 & .6471 & 0.941 & 26 & 5144 & 427 & 30 & 280 & & 4454 & \\
\hline $\mathrm{D}$ & 21 & 993 & 4.3 & 1110 & .7387 & 0.543 & 78 & 103 & 479 & 31 & 503 & & 8850 & \\
\hline $\bar{E}$ & 563 & 108 & 3.3 & 0.1364 & 24.1934 & 0.877 & 450 & 6427 & 2459 & 33 & .1196 & 0767 & 5557 & 86 \\
\hline $\bar{F}$ & 944 & 9.299 & 3.2 & 0.1625 & 19.6923 & 0.795 & 0.520 & .5882 & 2600 & 35 & .1292 & .0760 & 4649 & 9.77 \\
\hline $\bar{G}$ & 236 & 11.426 & 4.5 & 0.1589 & 28.3197 & 0.869 & 0.715 & 4828 & 2934 & 39 & .1381 & .0667 & 3638 & 11.262 \\
\hline $\mathrm{H}$ & 4.269 & 12.585 & 4.0 & 0.1750 & 22.8571 & 0.869 & 0.700 & 0.5659 & 0.3367 & 37 & 0.1521 & 0.0861 & 4320 & 11.213 \\
\hline I & 4.802 & 12.832 & 3.3 & 0.1979 & 16.6751 & 0.795 & 0.653 & 0.5728 & 3179 & 33 & 0.1573 & 0.0901 & .4112 & 11.095 \\
\hline $\mathrm{J}$ & 5.092 & 13.029 & 4.9 & 0.1260 & 38.8889 & 1.235 & 0.617 & 0.6593 & 3460 & 31 & 0.1556 & .1026 & 5931 & 9.5 \\
\hline $\bar{K}$ & 5.316 & 13.181 & 4.8 & 0.1622 & 29.5931 & 0.727 & 0.779 & 0.4919 & 3260 & 33 & 0.1179 & .0580 & 0.3900 & 8.8 \\
\hline $\mathrm{L}$ & 5.566 & 13.409 & 4.6 & 0.1778 & 25.8718 & 0.733 & 0.818 & 093 & 3540 & 38 & 303 & 0.0664 & 357 & 10.6 \\
\hline$\overline{\mathrm{M}}$ & 5.839 & 13.528 & 5.5 & 0.1654 & 33.2527 & 0.625 & & 789 & & 45 & 33 & 95 & & 11.2 \\
\hline $\mathrm{N}$ & 6.003 & 13.671 & 4.3 & 0.1525 & 28.1967 & 1.632 & 0.656 & 0.6836 & 10 & 40 & 0.2489 & 01 & 5590 & $\overline{15.50}$ \\
\hline$\overline{\mathrm{O}}$ & 6.242 & 14.823 & 4.2 & 0.1900 & 22.1053 & 0.854 & 798 & 0.5789 & 3900 & 411 & 0.1623 & .0940 & 3107 & 12.83 \\
\hline $\mathrm{P}$ & 452 & 15.071 & 6.3 & 1075 & 58.6047 & 0.681 & 677 & 6261 & 604 & 39 & 0.0732 & .0458 & 6098 & 8.1 \\
\hline $\bar{Q}$ & 6.719 & 15.413 & 4.7 & 1379 & 34.0827 & 0.649 & 0.648 & 0.6667 & 3670 & 36 & 0.0895 & 0.0597 & .5733 & $\overline{8.36}$ \\
\hline $\mathrm{R}$ & 6.896 & 18.287 & 3.9 & 0.2172 & 17.9558 & 1.213 & 0.847 & 0.5182 & 3700 & 34 & 0.2635 & 0.1365 & .3551 & $\overline{13.56}$ \\
\hline $\mathrm{S}$ & 343 & 3.579 & 5.8 & 0.1661 & 34.9187 & 0.869 & 0.963 & 50 & 20 & 30 & 0.1443 & 43 & 35 & 8.8 \\
\hline $\mathrm{T}$ & 7.536 & 18.706 & 4.8 & 0.2005 & 23.9402 & 0.625 & 0.962 & 13 & 50 & 24 & 0.1253 & 0.0666 & 789 & 6.6 \\
\hline $\mathrm{U}$ & 7.785 & 18.935 & 5.0 & 0.1994 & 25.0752 & 0.916 & 0.997 & 0.5339 & 0.4520 & 25 & 0.1827 & 0.0975 & 3818 & 8.30 \\
\hline$\overline{\mathrm{V}}$ & 8.024 & 19.020 & 5.6 & 0.1745 & 32.0917 & 0.839 & 0.977 & 0.5432 & \begin{tabular}{l|l}
0.4510 \\
\end{tabular} & 28 & 0.1464 & 0.0795 & 4152 & 8.325 \\
\hline$\overline{\mathrm{W}}$ & 8.355 & 21.161 & 7.6 & 0.1179 & 64.4614 & 0.754 & 0.896 & 0.6124 & \begin{tabular}{l|l|}
0.4660 \\
\end{tabular} & 30 & 0.0889 & 0.0544 & 5695 & 6.950 \\
\hline$X$ & 9.130 & 22.447 & 5.8 & 0.1788 & 32.4385 & 1.072 & 1.037 & 0.5469 & 0.4820 & 25 & 0.1917 & 0.1048 & 0.4130 & 8.50 \\
\hline $\bar{Y}$ & 9.340 & 22.593 & 4.7 & 0.2072 & 22.6834 & 1.017 & 0.974 & 0.5514 & 0.4560 & 27 & 0.2107 & 0.1162 & 0.3868 & 9.63 \\
\hline Z & 9.730 & 22.800 & 4.8 & 0.2155 & 22.2738 & 0.824 & 1.034 & 0.5432 & 0.4776 & 17 & 0.1776 & 0.0965 & 0.3737 & 6.5 \\
\hline
\end{tabular}

Source: Field survey, 1997 \& Statistical calculation

Table 3: Morphometric parameters for two river basins.

\begin{tabular}{|l|c|c|}
\hline \multicolumn{1}{|c|}{ Parameters } & Palung River & Chitlang River \\
\hline Total basin area $\left(\mathrm{km}^{2}\right)$ & 65.100 & 22.800 \\
\hline Basin perimeter $(\mathrm{km})$ & 37.734 & 21.345 \\
\hline Main stream length $(\mathrm{Km})$ & 15.950 & 9.730 \\
\hline Basin length $(\mathrm{km})$ & 13.540 & 8.070 \\
\hline Basin width $(\mathrm{km})$ & 4.810 & 2.830 \\
Basin slope $(\mathrm{Sb})$ & 0.467 & 0.493 \\
\hline Differences in elevation (m.) & 1030 & 1015 \\
\hline Basin shape $(\mathrm{Rf})$ & 0.355 & 0.350 \\
\hline Relief ratio $(\mathrm{Rr})$ & 0.076 & 0.126 \\
\hline Mean stream slope $(\mathrm{Sc})$ & 0.065 & 0.104 \\
\hline Sinuosity Index $(\mathrm{Si})$ & 1.347 & 1.278 \\
\hline Drainage density $(\mathrm{Dd})$ & 5.084 & 4.119 \\
\hline Drainage frequency $(\mathrm{Fd})$ & 14.316 & 8.728 \\
\hline Elongation ratio $(\mathrm{Re})$ & 0.194 & 0.256 \\
\hline Circularity ratio $(\mathrm{Rc})$ & 0.575 & 0.629 \\
\hline Compactness coefficient $(\mathrm{Cc})$ & 1.319 & 1.261 \\
\hline Ruggedness number $(\mathrm{HD})$ & 5.240 & 4.180 \\
\hline
\end{tabular}

Source: Field survey \& Topographic Maps (complied with application of GIS) 


\section{RESULTS AND DISCUSSION}

Relationship between stream slope and stream discharge for both rivers is obtained by the regression modeling as presented in following equations:

For Palung River, $\mathrm{S}=1.2469 \mathrm{Q}^{0.1757}$

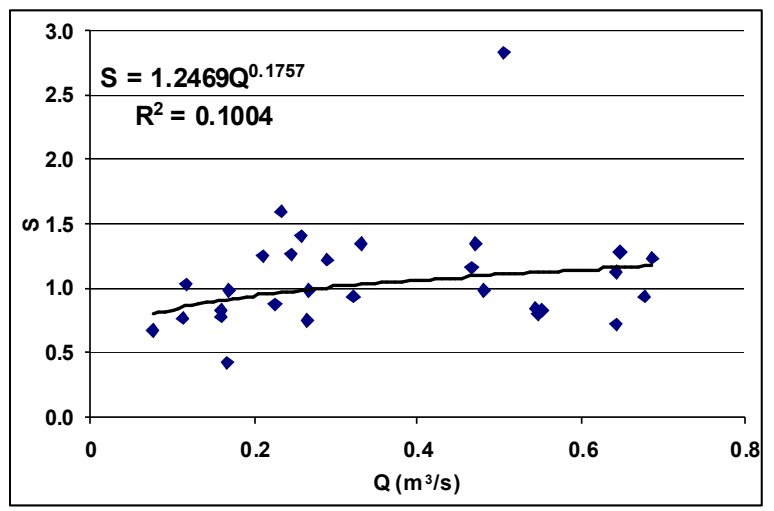

Figure 2a: Stream slope in Palung River

For Chitlang River, $\mathrm{S}=1.0345 \mathrm{Q}^{0.1923}$

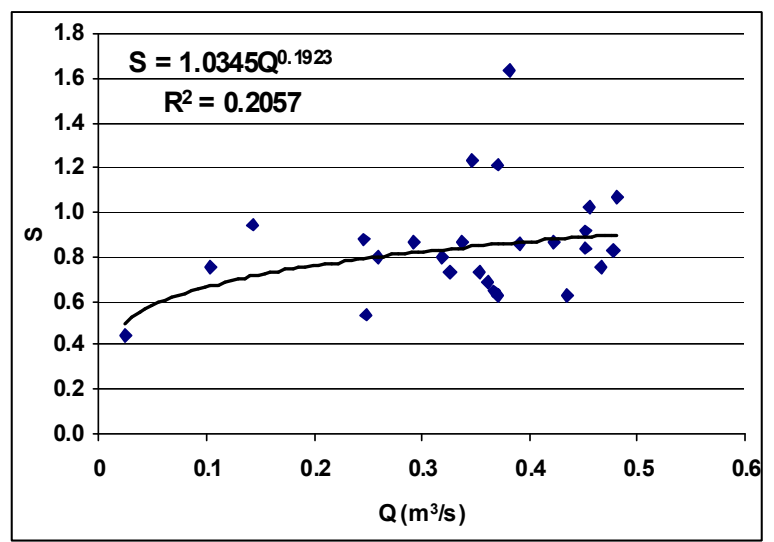

Figure 2b: Stream slope in Chitlang River

Water discharge increases in both channels in downstream direction; it ranges $0.075 \mathrm{~m}^{3} / \mathrm{sec}$ to $0.687 \mathrm{~m}^{3} / \mathrm{sec}$ along Palung River and $0.024 \mathrm{~m}^{3} / \mathrm{sec}$ to $0.482 \mathrm{~m}^{3} / \mathrm{sec}$ along Chitlang River. It is seen that the stream slope also increases in downstream direction for both rivers.
Similarly, relationships among the different parameters of flow hydraulics are the following.

Palung River

$\mathrm{T}_{\mathrm{c}}=4.9177 \mathrm{~d}^{-0.5961}$

$\mathrm{W}_{\mathrm{c}}=4.9626 \mathrm{~d}^{-0.7334}$

$\mathrm{V}_{\mathrm{c}}=0.5873 \mathrm{~d}^{-0.0441}$

\section{Chitlang River}

$\mathrm{T}_{\mathrm{c}}=0.6074 \mathrm{~d}^{-0.2712}$

$\mathrm{W}_{\mathrm{c}}=0.2300 \mathrm{~d}^{-0.2052}$

$\mathrm{V}_{\mathrm{c}}=0.3777 \mathrm{~d}^{-0.1159}$

Where, $T_{c}=$ boulder shear stress

$$
\begin{aligned}
& \mathrm{W}_{\mathrm{c}}=\text { stream power } \\
& \mathrm{V}_{\mathrm{c}}=\text { mean velocity }
\end{aligned}
$$

Particle's size decreases in downstream direction along both channels but there are abnormal changes in particle size in some sections. Intermediate diameter ranges $10 \mathrm{~cm}$ to $58 \mathrm{~cm}$ in Palung River and 17 to $31 \mathrm{~cm}$ in Chitlang River.

Figure $3 \mathrm{a}$ indicates that there is abnormal change in particle size near the $5^{\text {th }}$ and $13^{\text {th }} \mathrm{km}$ distance from origin along the Palung River. Figure $3 b$ indicates that there is abnormal change in particle size near the $3^{\text {rd }}, 5^{\text {th }}$ and $7^{\text {th }} \mathrm{km}$ distance from origin along the Chitlang River in downstream direction due to local obstacle of landslide and irrigational dam construction. Figure 4 represents the changing scenario of particle sizes along the main channels in downstream direction.

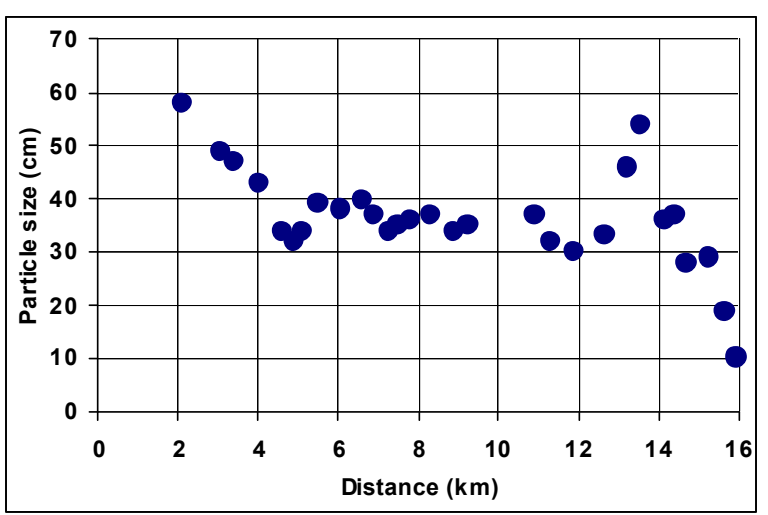

Figure 3a: Particle size in Palung River 


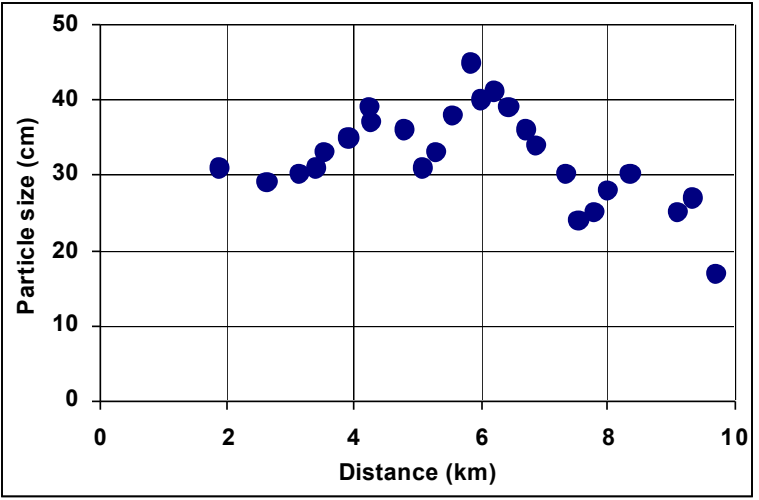

Figure 3b: Particle size in Chitlang River
The channel size changes in downstream direction in the both channels, where the width along Palung River ranges from $1.2 \mathrm{~m}$ to $10.6 \mathrm{~m}$ and mean depth ranges from $0.06 \mathrm{~m}$ to $0.35 \mathrm{~m}$. The width along Chitlang River ranges from $1.2 \mathrm{~m}$ to $5.3 \mathrm{~m}$ and mean depth ranges from $0.05 \mathrm{~m}$ to $0.22 \mathrm{~m}$. Capacity (cross-sectional area) in Palung River ranges from $0.2 \mathrm{~m}^{2}$ to $2.8 \mathrm{~m}^{2}$ and in Chitlang River it ranges from $0.07 \mathrm{~m}^{2}$ to $1.04 \mathrm{~m}^{2}$. Figure 5 shows the Channel cross-section for both rivers and figure 6 shows the hypsometric curve for both rivers.

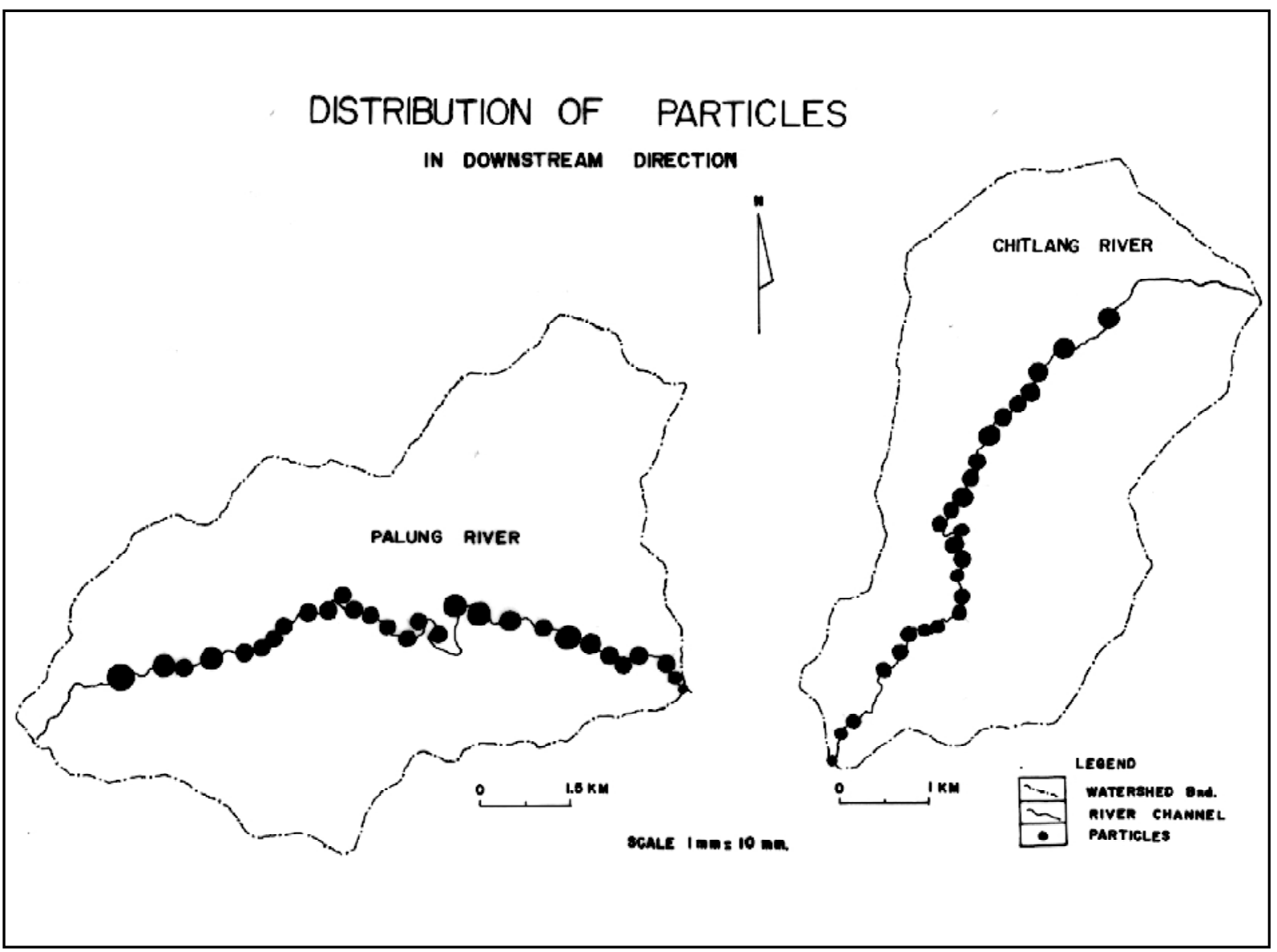

Figure 4: Distribution of particles in downstream direction 


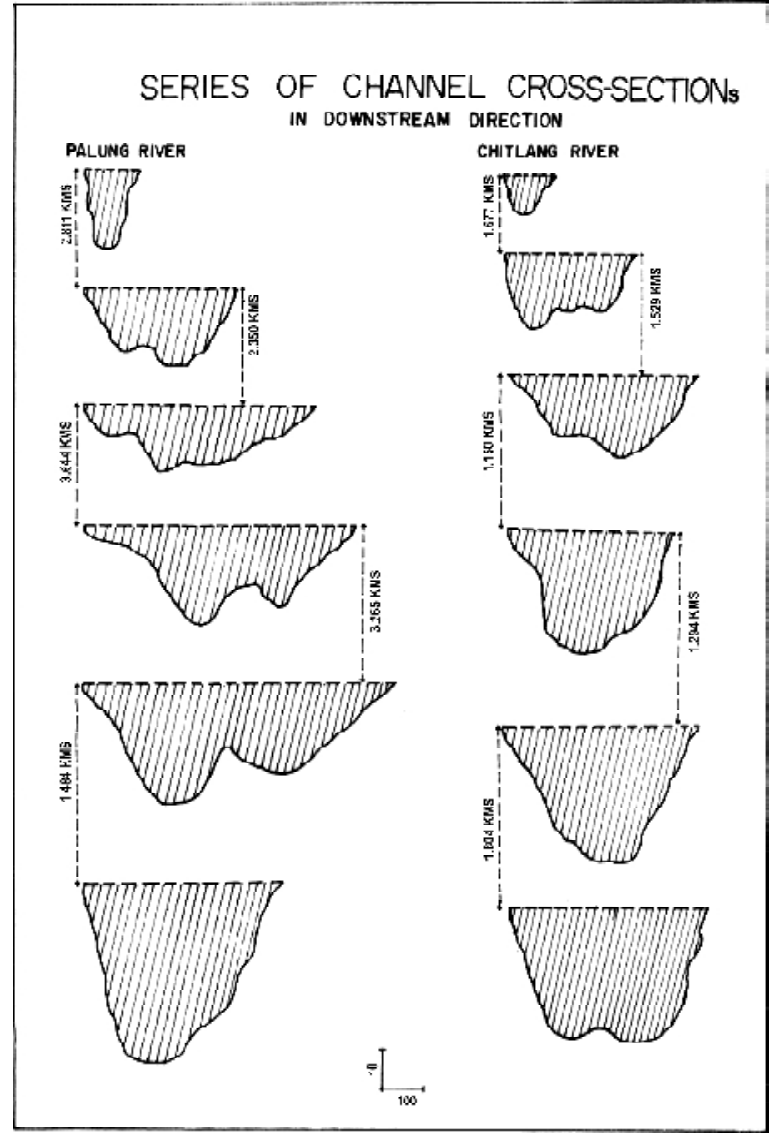

Figure 5: Series of channel cross-sections in downstream direction

\section{CONCLUSION}

Though there are abnormal changes in different parameters such as stream slope and particle size along downstream distance, water discharge is increasing with increasing in downstream distance of channel and particle's size is decreasing with increasing downstream distances. Hence, it is concluded that these streams are similar in their dynamics and form to streams in other parts of the World. Further, it is noted that present environment is characterized by one of the high relief, tectonic activity and intensive land use. This provides a great volume of both water and sediment to the channels which forced them to behave in an abnormal way.

Journal of Hydrology and Meteorology, Vol.6, No. 1
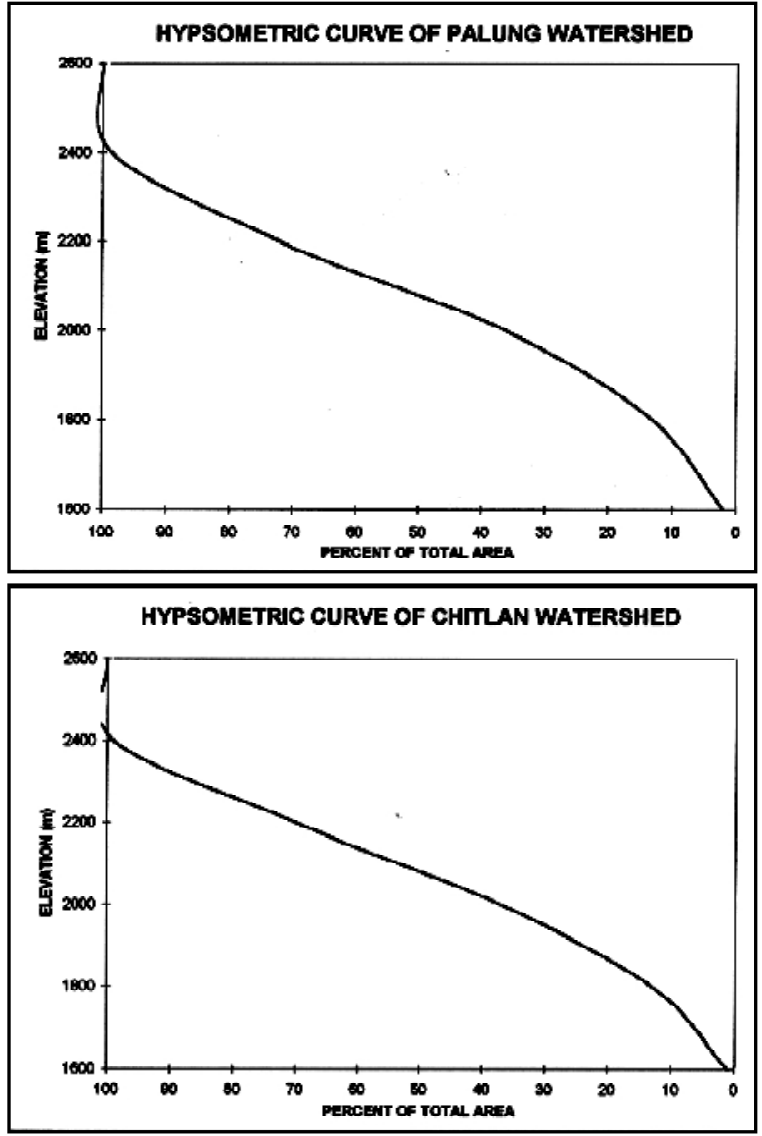

Figure 6: Hypsometric curve of Palung and Chitlang Rivers.

Some of the physical controls i.e. geological structure on channel bed and landslide contribute large boulder to the channels which caused irregular change in particular size. Other human factors also play important role in creating irregularities in channel hydraulics and water and sediment discharge. These include division of water for irrigation and water mills, abstraction of gravels and rocks from the river bed and other river training activities in this area.

\section{ACKNOWLEDGEMENT}

The author wishes to sincerely thank Dr. Dilip Kumar Gautam, Senior Divisional Hydrologist, Department of Hydrology and Meteorology for his valuable suggestions and guidance in this paper. 


\section{REFERENCES}

Alford, D., 1991. Hydrological Aspect of Himalayan Region, Occasional Paper No. 18, Kathmandu, Nepal: ICIMOD.

Caine, M., and Mool, P.K, 1981. Channel Geometry and flow estimates for two small Mountain streams in the Middle Hills of Nepal, Mountain Research and Development, Vol. 1, No. 3-4, 1981.

Chorley, R.J., Schuman, S.A. and Sugdan, D.E, 1984. Geomorphology, Methuen, London.

Chow, V.T., 1986. Open Channel Hydraulics, McGraw-HillCompany, London, pp.19-82.

DHM, 1997. Stream flow Records of Nepal, Department of Hydrology \& Meteorology, Kathmandu, Government of Nepal, 1963-78.

DHM, 1997. Climatological Record Books of Nepal, Department of Hydrology \& Meteorology, Kathmandu, Government of Nepal, 1971-90.

Gardiner, V., and Dackombe, R.,1983. Geomorphological Field Mannual, George Allen \& Unwin(publisher) Ltd., London.

Goudie,A., 1990. Geomorphological Technique, University of Oxford, London, 1990.

Gregory, S., 1968. Statistical methods and Geographers, Longman, Green and Company Ltd., London.

Johnson, R., and Collins, R, 1996. Development in a methodology of classifying sediments in Himalayan Rivers, Proceeding of the International Conference on Ecohydrology of High Mountain Areas, Kathmandu, Nepal.
Khanal, N.R., 1992. Study of Geo-hydrology, Landuse and Population of the Makalu Barun Conservation Project Area, The Makalu-Barun Conservation Project, Working Paper Publication Series, Report 14.

Leopold, L.B., and Maddock, T, 1953. The hydraulic geometry of stream channels and some physiographical implication, US Geological Survey Professional Paper, No. 252.

Strahler, A.N., 1968. Introduction to Physical Geography, Third Edition, John Willey and Sons, Inc., New York. 